\title{
The Modification of the Flavonoid Naringenin by Bradyrhizobium sp. Strain ORS285 Changes the nod Genes Inducer Function to a Growth Stimulator
}

\author{
Nico Nouwen, ${ }^{1,+}$ Daniel Gargani, ${ }^{2}$ and Eric Giraud ${ }^{1}$ \\ ${ }^{1}$ IRD, Laboratoire des Symbioses Tropicales et Méditerranéennes, UMR IRD/SupAgro/INRA/UM2/CIRAD, Montpellier, France \\ ${ }^{2}$ CIRAD, UMR BGPI, Montpellier, France \\ Accepted 30 June 2019.
}

\begin{abstract}
As inducers of nodulation (nod) genes, flavonoids play an important role in the symbiotic interaction between rhizobia and legumes. However, in addition to the control of expression of nod genes, many other effects of flavonoids on rhizobial cells have been described. Here, we show that the flavonoid naringenin stimulates the growth of the photosynthetic Bradyrhizobium sp. strain ORS285. This growth-stimulating effect was still observed for strain ORS285 with nodD1, nodD2, or the naringenindegrading $f d e$ operon deleted. Phenotypic microarray analysis indicates that in cells grown in the presence of naringenin, the glycerol and fatty acid metabolism is activated. Moreover, electron microscopic and enzymatic analyses show that polyhydroxy alkanoate metabolism is altered in cells grown in the presence of naringenin. Although strain ORS285 was able to degrade naringenin, a fraction was converted into an intensely yellowcolored molecule with an $\mathrm{m} / \mathrm{z}(+)$ of 363.0716 . Further analysis indicates that this molecule is a hydroxylated and $\mathrm{O}$-methylated form of naringenin. In contrast to naringenin, this derivative did not induce nod gene expression, but it did stimulate the growth of strain ORS285. We hypothesize that the growth stimulation and metabolic changes induced by naringenin are part of a mechanism to facilitate the colonization and infection of naringeninexuding host plants.
\end{abstract}

Keywords: flavonoid, mutualism, nodule organogenesis, rhizobium

Legumes are able to establish a symbiotic interaction with nitrogen-fixing bacteria, called rhizobia, which allows them to grow in nitrogen-poor soils. This symbiotic nitrogen fixation has a high impact in natural environments, as well as in agriculture environments, as it is one of the largest contributors of nitrogen input in soil. The symbiotic interaction between legumes and rhizobia depends on a molecular dialogue between both partners, in which plant-exuded flavonoids have been shown to play distinct roles. They are rhizobial growth

${ }^{\dagger}$ Corresponding author: N. Nouwen; nico.nouwen@ird.fr

Funding: This work was supported financially by the Agence Nationale de la Recherche, grant SymEffectors number ANR-16-CE20-0013.

*The $e$-Xtra logo stands for "electronic extra" and indicates that seven supplementary figures are published online.

The author(s) declare no conflict of interest.

() 2019 The American Phytopathological Society stimulators and chemoattractants, they induce the expression of nodulation (nod) genes in compatible rhizobia, they determine host specificity, they control root development, and they modify plant defense responses (Hassan and Mathesius 2012).

The basic chemical structure of flavonoids is composed of a 15-carbon skeleton arranged in three rings: two benzene rings ( $\mathrm{A}$ and $\mathrm{B}$ ) that are connected via a pyran ring containing an oxygen atom ( $\mathrm{C}$ ring) (Fig. 1). Depending on the carbon in the $\mathrm{C}$ ring on which the $\mathrm{B}$ ring is attached and on substitutions on and the oxidation level of the $\mathrm{C}$ ring, flavonoids can be divided into different subgroups, whereas additions of hydroxyl, methoxyl, and other substituents to the A and B rings are characteristic for individual members. Subgroups of flavonoids include flavones, flavonols, flavonones, isoflavones, anthocyanins, and chalcones (Panche et al. 2016).

In rhizobium-legume symbiosis, flavonoid-induced nod gene expression has been well studied. Expression of nod genes is regulated by NodD transcriptional regulators that are members of the LysR family of transcriptional regulators. Some studies have suggested that flavonoids directly interact with NodD (Li et al. 2008; Yeh et al. 2002), but despite many years of research, experiments showing an indisputable binding of flavonoids to NodD proteins are still lacking. Induction of the nod genes in rhizobia leads to the synthesis and secretion of lipochitooligosaccharides, or Nod factors (NFs). Once NFs are perceived by the host plant, a signaling pathway is activated that leads to the formation of nodules and allows the infection of the plant tissue by the rhizobia. Remarkably, addition of specific flavonoids to the rhizobium strain Azorhizobium caulinodans ORS571 has also been shown to promote intercellular root colonization of Arabidopsis thaliana, rice, and wheat roots, and most importantly, this colonization has been shown to be independent of nod gene expression (Gough et al. 1997; Jain and Gupta 2003; Webster et al. 1997).

Several rhizobia have been shown to catabolize nod geneinducing flavonoids (Cooper et al. 1995; Gajendiran and Mahadevan 1988; Rao and Cooper 1994). Via analysis of the formed cleavage products, the degradation pathways for several flavonoids in rhizobia have been reconstructed (Cooper et al. 1995). In rhizobia, the degradation of flavonoids seems to start with a Cring fission that, thereby, releases the $\mathrm{A}$ and $\mathrm{B}$ rings. Degradation of different flavonoids often results in common intermediates such as phloroglucinol and protocathechuic acid. Although these experiments have led to a pathway for the degradation of a number of flavonoids in rhizobia, the genes implicated in these degradation pathways, at the time of this research, were not known. Recently, a cluster of 11 genes (fde operon) has been shown to be implicated in the degradation of the flavonoid 
naringenin in the endophytic bacterium Herbaspirillum seropedicae SmR1 (Marin et al. 2013). A similar gene cluster has been found in several Bradyrhizobium sp. strains (Marin et al. 2013).

Certain photosynthetic bradyrhizobia are able to establish an NF-independent symbiosis with some tropical aquatic legumes of the Aeschynomene genus (Arrighi et al. 2012; Giraud et al. 2007). It remains unknown whether flavonoids play an important role during this NF-independent symbiosis. During our previous research, we made the observation that addition of root exudates of host plants or pure flavonoids such as naringenin and apigenin (Fig. 1A and B, respectively) results in a significant increase in the optical density (OD) of cultures from the photosynthetic Bradyrhizobium sp. strains ORS278 and ORS285 (Nouwen et al. 2016). In this study, we investigated the observed increase in growth rate induced by the flavonoid naringenin in more detail. As a model strain, we used strain ORS285, which in contrast to strain ORS278 does have the canonical nodABC genes and is able to initiate an NFdependent or NF-independent symbiosis, depending on the host plant (Giraud et al. 2007). The nodABC genes in strain ORS285 are induced by the flavonoids naringenin and apigenin and are absolutely required for symbiosis with $A$. afraspera (Nouwen et al. 2016). However, the nodABC genes can be deleted without impacting symbiosis with Aeschynomene spp. such as A. indica and A. evenia (Arrighi et al. 2012; Giraud et al. 2007). Here, we studied the effects of different carbon sources, the individual roles of the symbiotic regulators NodD1 and NodD2, and the effect of deletion of the operon implicated in naringenin degradation (fde operon) on the growthstimulating effect of naringenin. The data indicate that this growth stimulation does not depend on the symbiotic regulators NodD1 or NodD2 and that naringenin degradation is not implicated in the observed effect. Moreover, we show that part of the naringenin is not degraded but is converted into a molecule that is unable to induce nod gene expression but that is able to stimulate growth of strain ORS285.

\section{RESULTS}

\section{Growth of strain ORS285 in the presence of different naringenin concentrations.}

Previously, we have shown that addition of certain flavonoids or root exudates of host plants to the culture medium results in a significant increase in the culture density of strain ORS285 (Nouwen et al. 2016). To investigate this phenomenon in more detail, we determined the growth curve of strain ORS285 in the presence of different concentrations of the nod gene-inducing flavonoid naringenin. At early time points on the growth curve, a higher naringenin concentration resulted in a faster increase in the OD of the cell culture (Fig. 2A through G). However, whereas at low naringenin concentrations (125 nM and $250 \mathrm{nM}$ ) growth began after a long lag phase, the final OD of cell cultures obtained was almost twofold higher compared with cultures grown in the presence of high naringenin concentrations ( $\geq 500 \mathrm{~nm}$ ) (Fig. $2 \mathrm{~A}$ and $\mathrm{B}$ versus $\mathrm{C}$ through $\mathrm{G}$ ). To control whether the observed increase in OD reflects an

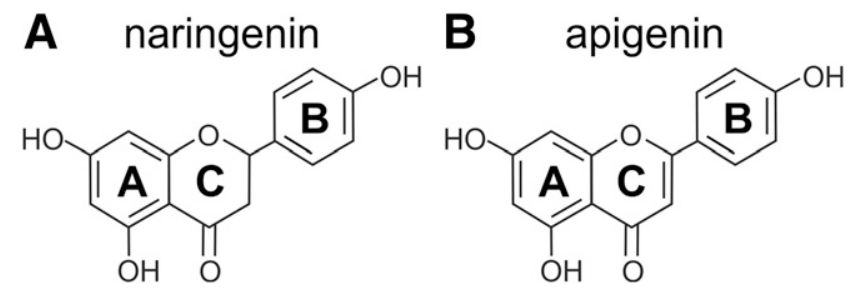

Fig. 1. Chemical structures of $\mathbf{A}$, naringenin and $\mathbf{B}$, apigenin flavonoids used in this study. increase in the number of bacteria, we determined the number of CFU at the end of the growth curve of three representative cultures. This showed that the CFU of the culture grown in the presence of $5 \mu \mathrm{M}$ naringenin was approximately twice as high as in the culture grown in the absence of naringenin and around twofold lower than the culture grown in the presence of $250 \mathrm{nM}$ naringenin (Fig. 2H). The OD of the three cultures was approximately 1.5 , approximately 0.75 , and approximately 3.0 , respectively (Fig. 2A, B, and F), which indicated that the OD of the culture medium correlated well with the number of $\mathrm{CFU}$ present in the culture. In the previous experiment, naringenin was present from the start of the experiment. To analyze whether naringenin could also stimulate the growth of a culture that had already been initiated in minimal medium, we grew strain ORS285 for 3 days in the absence of naringenin and subsequently added it to one half of the culture (Fig. 3A). The OD of this culture increased more rapidly than the culture to which no naringenin was added, and the number of CFU at the end of the experiment was approximately three- to fourfold as high (Fig. 3B). This indicates that naringenin addition stimulates the growth of an already growing culture.

\section{Effect of NodD mutations and carbon and nitrogen source on naringenin-dependent growth stimulation.}

It has previously been shown that naringenin induces the expression of nod genes in strain ORS285 and that the regulators NodD1 and NodD2 play an important role in the expression of the nodA-J operon (Nouwen et al. 2016; Renier et al. 2011). To analyze whether one of the two NodD regulators modulates the naringenin-dependent growth stimulation, we determined the growth curves of strain ORS285 $\Delta$ nodD1 and $\triangle$ nodD2 mutants (Fig. 4A and B, respectively). The growth of both nodD mutants was stimulated by the addition of naringenin.

The observed increase in growth rate indicated that addition of naringenin in some way affected the overall cell physiology of strain ORS285 cells. To determine whether the culture conditions had an impact on the growth stimulation by naringenin, we grew strain ORS285 in minimal medium containing different carbon and nitrogen sources. With all tested carbon and nitrogen sources, $5 \mu \mathrm{M}$ naringenin increased the growth rate of strain ORS285 (Fig. 5). Interestingly, whereas growth of strain ORS285 in minimal medium containing glycerol as the carbon source was not detectable in the timeframe of the experiment ( 5 days), slow but substantial growth was observed when the culture medium contained $5 \mu \mathrm{M}$ naringenin (Fig. 5G). Taken together, we demonstrated that naringenin stimulates the growth of strain ORS285 and that this growth stimulation is independent of the carbon or nitrogen substrate in the culture medium and is not impacted by deletion of one of the symbiotic regulators NodD1 or NodD2.

\section{Phenotyping of strain ORS285 cells grown in the absence and presence of naringenin.}

To investigate whether specific metabolic pathways are activated in the presence of naringenin, we performed a phenotypic microarray analysis using GN2 MicroPlates (Biolog, Hayward, CA, U.S.A.). In this assay, substrates metabolized by the bacterium produce NADH from which electrons reduce a tetrazolium dye, resulting in a purple color. To this end, strain ORS285 pregrown in the absence and presence of $5 \mu \mathrm{M}$ naringenin was distributed into the wells of GN2 MicroPlates, and color development in the plates was followed for 3 days. In general, with substrates that lead to dye reduction under both conditions, the color development for cells grown in the presence of naringenin was faster compared with nontreated cells (Supplementary Fig. S1). However, the most remarkable 
observation was that nontreated cells were unable to reduce the tetrazolium dye with glycerol and the detergents TWEEN 40 and TWEEN 80 (Sigma-Aldrich, St. Louis) used as substrate, whereas cells grown in the presence of naringenin could reduce the tetrazolium dye (Fig. 6). These results suggest that the glycerol and fatty acid metabolism was activated in cells grown in the presence of naringenin.

\section{Electron microscopic analysis of cells grown} in the presence of naringenin.

To analyze the morphology of strain ORS285 grown in the presence of $5 \mu \mathrm{M}$ naringenin, cells that had been grown on succinate-glutamate minimal medium plates were analyzed by transmission electron microscopy (TEM). Cells grown in the absence and presence of naringenin had a similar morphology (Fig. 7A and B; Supplementary Fig. S2A and B). However, there was an important difference in the size and number of granule-like structures present in the cytoplasm of the cells. The cytoplasm of cells grown in the absence of naringenin contained many small granules (Fig. 7A). In contrast, cells grown in the presence of naringenin contained fewer but larger granules (Fig. 7B). These structures likely represent polyhydroxyalkanoate (PHA) granules, a lipid storage polymer frequently found in rhizobia. To analyze whether naringenin addition indeed affects PHA metabolism, we determined the 3hydroxybutyrate dehydrogenase (BdhA) (EC 1.1.1.30) activity
A

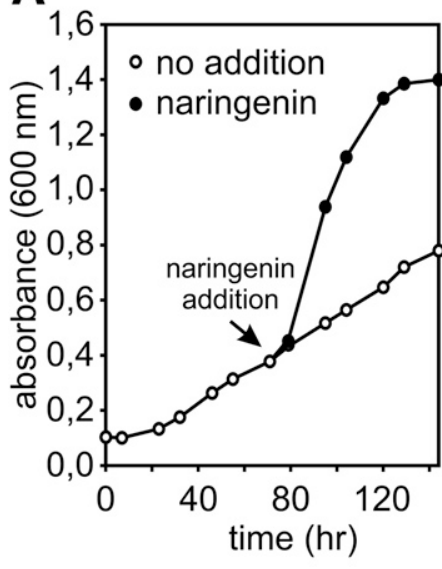

B

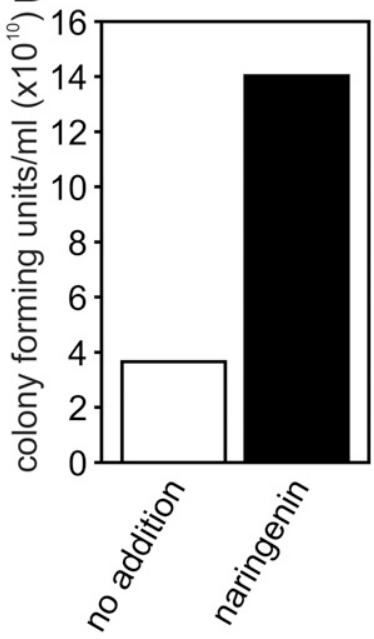

Fig. 3. Effect of naringenin addition after 3 days of growth on minimal buffered nodulation medium (BNM). A, Representative growth curve of Bradyrhizobium sp. strain ORS285 in minimal BNM and addition of $5 \mu \mathrm{M}$ naringenin after 3 days of growth. B, Bars indicate the number of CFU present in 6-day-old cultures of strain ORS285 as shown in panel A.

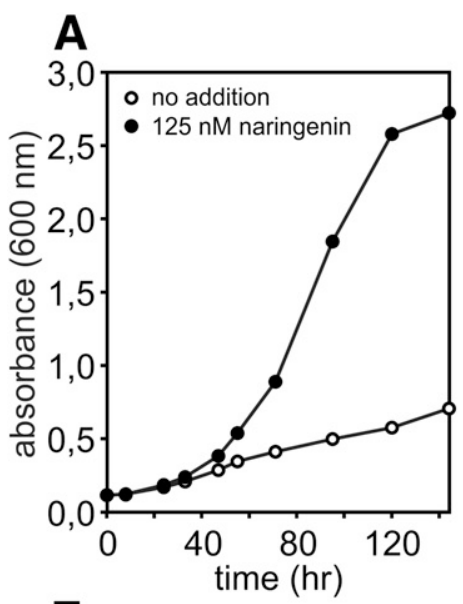

E

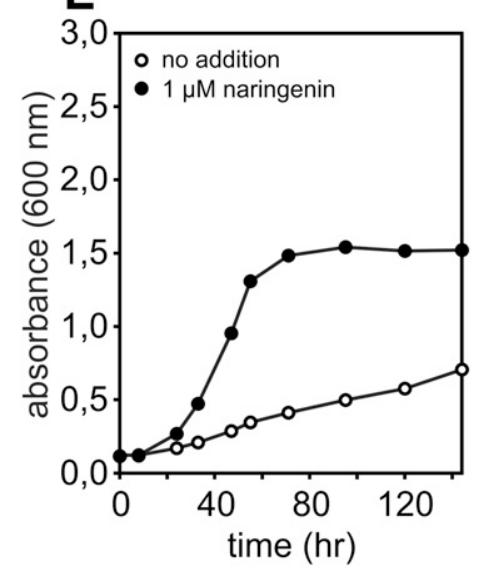

B

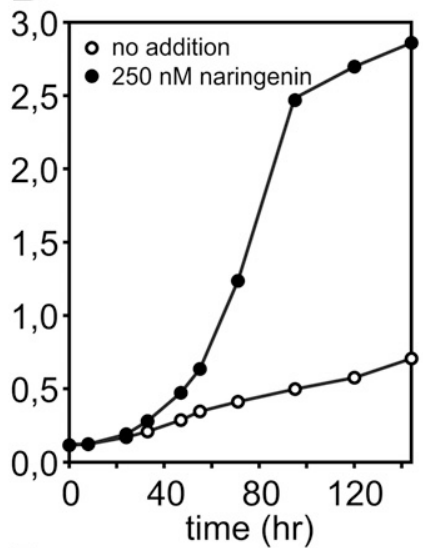

$\mathbf{F}$

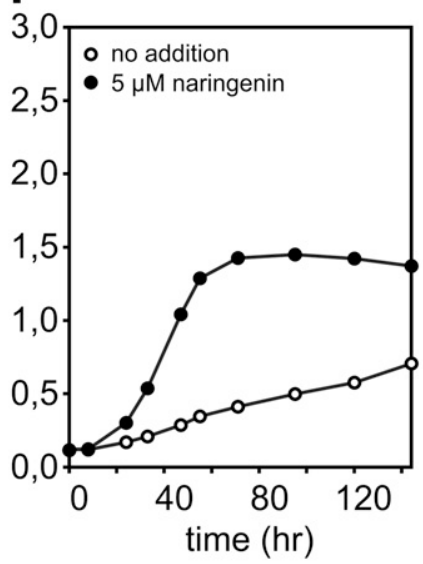

C

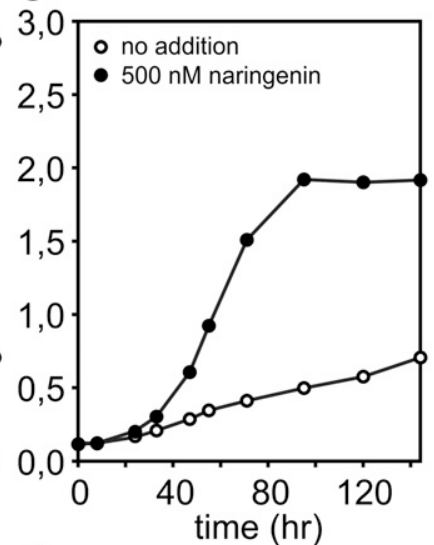

G

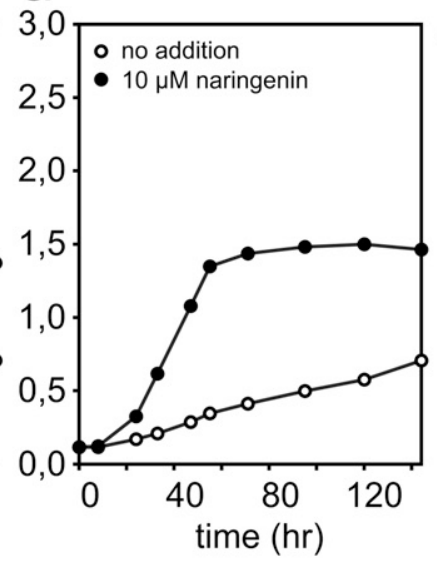

D

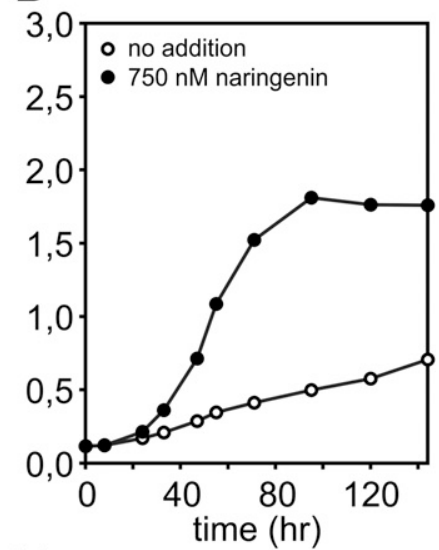

$\mathrm{H}$

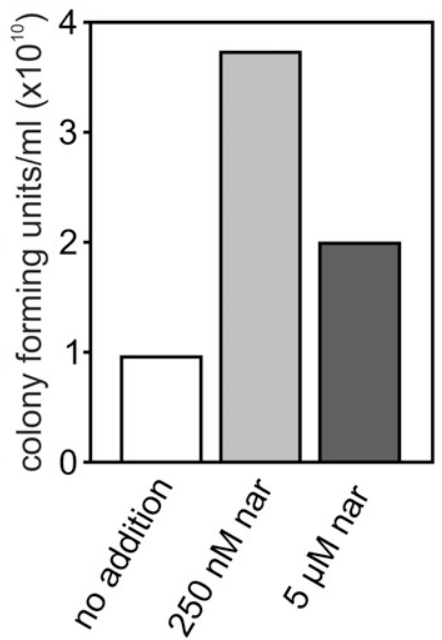

Fig. 2. Representative growth curves and number of CFU of Bradyrhizobium sp. strain ORS285 grown in the presence of different concentrations of naringenin. Growth of strain ORS285 cultivated in minimal buffered nodulation medium in the absence and presence of naringenin at concentrations of $\mathbf{A}, 125 \mathrm{nM}$; $\mathbf{B}$, 250 $\mathrm{nM}$; C, $500 \mathrm{nM}$; D, $750 \mathrm{nM} ; \mathbf{E}, 1 \mu \mathrm{M} ; \mathbf{F}, 5 \mu \mathrm{M}$; or $\mathbf{G}, 10 \mu \mathrm{M}$. H, Bars indicate the number of CFU present in 5-day-old strain ORS285 cultures grown in the absence and presence of $250 \mathrm{nM}$ and $5 \mu \mathrm{M}$ naringenin, respectively. 
in cells. This enzyme plays a very important role in the degradation of PHA granules (Trainer and Charles 2006). The enzyme activity in cells grown in the presence of naringenin was approximately 10-fold higher compared with the activity measured under normal conditions (Fig. 7C). Taken together, both TEM pictures of cells and measured BdhA activities

A
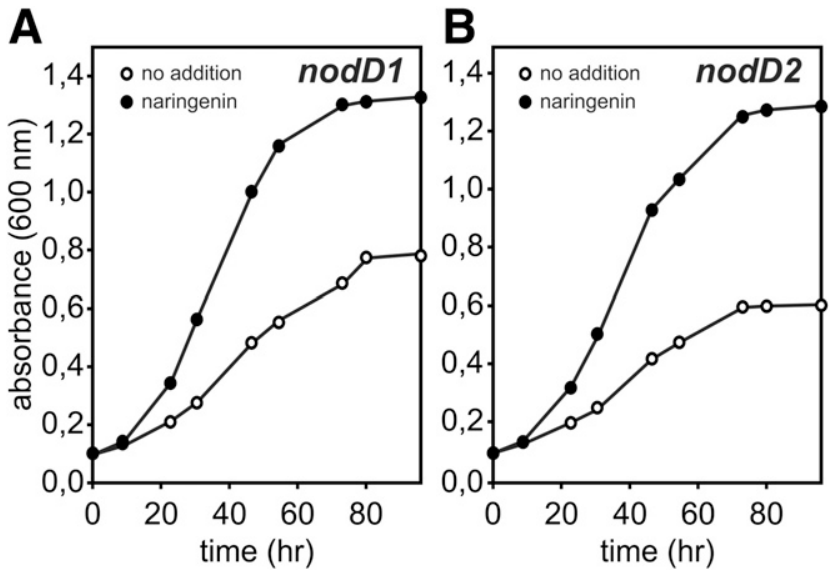

Fig. 4. Naringenin stimulates growth of Bradyrhizobium sp. strain ORS285 $\triangle$ nodD1 and $\triangle$ nodD2 mutants. Representative growth curves in the absence and presence of $5 \mu \mathrm{M}$ naringenin for A, Strain ORS285 $\Delta$ nodD1; and B, strain ORS285 $\Delta$ nodD2. demonstrated that naringenin addition has a strong impact on the PHA metabolism of strain ORS285 cells.

Naringenin and apigenin degradation by strain ORS285.

Due to the presence of two benzene rings and one pyran ring (Fig. 1), flavonoids have a typical absorbance spectrum, which can easily be detected using a spectrophotometer. Modification on or opening of one of the three rings of the flavonoid structure has a direct impact on the absorbance spectrum. To analyze whether strain ORS285 degrades naringenin and another nod gene-inducing flavonoid, apigenin, we determined the absorbance spectrum of the growth medium after different incubation time periods in the presence of $20 \mu \mathrm{M}$ naringenin and $20 \mu \mathrm{M}$ apigenin. In the presence of strain ORS285 cells, we observed that the absorbance spectrum of both naringenin- and apigenin-containing culture medium changed over time (Fig. $8 \mathrm{~A}$ and $\mathrm{C}$ ). Such changes in absorbance spectrum were not seen in the absence of strain ORS285, and culture medium with strain ORS285 grown in the absence of flavonoids did not have an absorbance spectrum similar to that found for cells grown in the presence of naringenin and apigenin (Supplementary Fig. $\mathrm{S} 3$ ). In H. seropedicae SmR1 an operon of 10 genes (fde operon) has been shown to be involved in the degradation of naringenin (Marin et al. 2013). Genome analysis showed that a similar operon is present in strain ORS285 (open reading frame accession numbers: BRAD285_V2_3671 through 3680; Fig. 8E). To analyze whether this operon is implicated in the observed change in absorbance spectrum, we constructed a
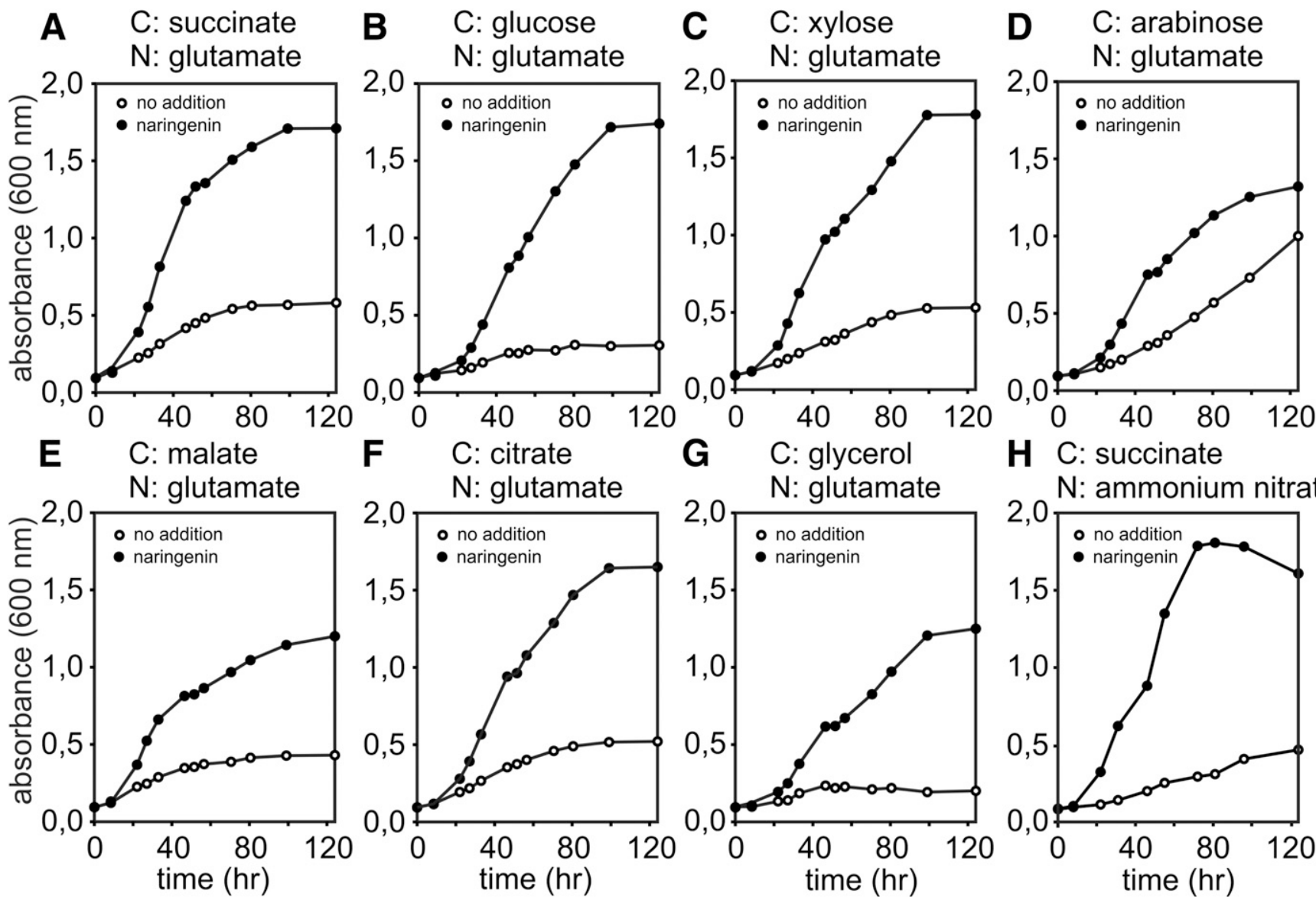

G

C: glycerol $\mathrm{N}$ : glutamate

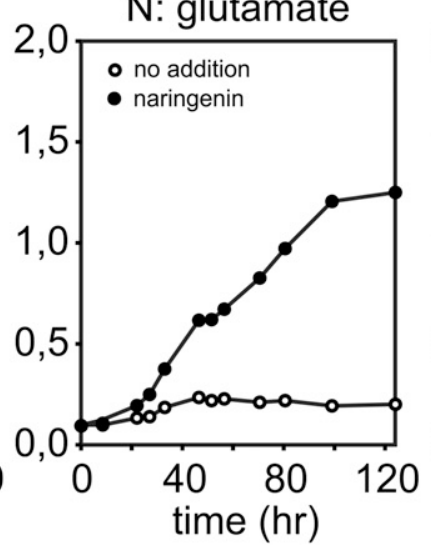

H C: succinate

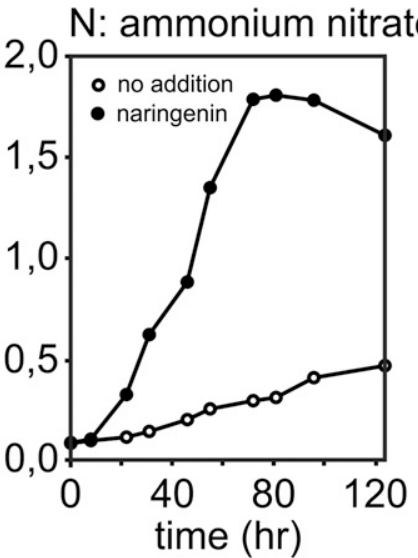

Fig. 5. Growth stimulation by naringenin is independent of the carbon and nitrogen source present in the culture medium. Representative growth curves of Bradyrhizobium sp. strain ORS285 cells grown in the absence and presence of $5 \mu \mathrm{M}$ naringenin in minimal buffered nodulation medium containing different sources of carbon and nitrogen: A, $10 \mathrm{mM}$ succinate and $5 \mathrm{mM}$ glutamate; B, $10 \mathrm{mM}$ glucose and $5 \mathrm{mM}$ glutamate; C, $10 \mathrm{mM}$ xylose and $5 \mathrm{mM}$ glutamate; D, $10 \mathrm{mM}$ arabinose and $5 \mathrm{mM}$ glutamate; $\mathbf{E}, 10 \mathrm{mM}$ malate and $5 \mathrm{mM}$ glutamate; $\mathbf{F}, 10 \mathrm{mM}$ citrate and $5 \mathrm{mM}$ glutamate; $\mathbf{G}$, $10 \mathrm{mM}$ glycerol and $5 \mathrm{mM}$ glutamate; or $\mathbf{H}, 10 \mathrm{mM}$ succinate and $5 \mathrm{mM}$ ammonium nitrate. 
mutant strain in which the complete operon has been deleted (ORS285 $\Delta f d e$; Fig. 8E). When strain ORS285 $\Delta$ fde was used, the absorbance spectrum of naringenin- and apigenincontaining culture medium did not change (Fig. 8B and D). This indicates that strain ORS285 can degrade naringenin and apigenin and that this requires the presence of the fde operon. Interestingly, growth experiments with strain ORS285 $\Delta f d e$ cells showed that naringenin addition still stimulated growth (Fig. 8G), whereas apigenin addition hardly affected the growth of strain ORS285 $\Delta$ fde cells (Fig. 8G). This indicates that the fde operon is required for apigenin-dependent growth stimulation, but not for naringenin-dependent growth stimulation.

\section{Culture medium of cells grown in the presence} of naringenin contains a molecule that stimulates growth.

The experiments described above show that naringenin is rapidly degraded by strain ORS285. However, the appearance of new absorption peaks in the absorbance spectrum and a remarkably yellow and time-dependent coloration of the growth medium (Supplementary Fig. S7A) indicate that after naringenin addition novel molecules are formed that remain present in the culture medium. Therefore, we questioned whether the observed growth stimulation is due to naringenin itself or to one of these newly formed molecules. To test this, we separated the culture supernatant from strain ORS285 that had been grown for 3 days in the presence of $5 \mu \mathrm{M}$ naringenin. After heat sterilization and dilution with fresh medium, this old culture supernatant was used to grow strain ORS285 (Fig. 9A and B). We observed that with the old culture supernatant strain ORS285 grew much faster. Moreover, under these conditions, no additive effect of naringenin on growth stimulation was observed (Fig. 9A and B). This indicates that the old culture supernatant of cells previously grown in the presence of naringenin contains a molecule or molecules that are capable of stimulating the growth of strain ORS285. To isolate these molecules from the culture supernatant, we developed a purification protocol using solid-phase extraction and subsequent high-performance liquid chromatography (HPLC) purification. Using this protocol, we obtained an intensely yellow HPLC fraction that when added to the culture medium was able to stimulate the growth of strain ORS285 cells (Fig. 9C). Interestingly, this fraction was also able to stimulate the growth of Bradyrhizobium sp. strain ORS278 but not growth of B. diazoefficiens USDA110 (Supplementary Fig. S4). The isolated HPLC fraction had an absorbance spectrum that was clearly different from the one obtained with pure naringenin (Fig.
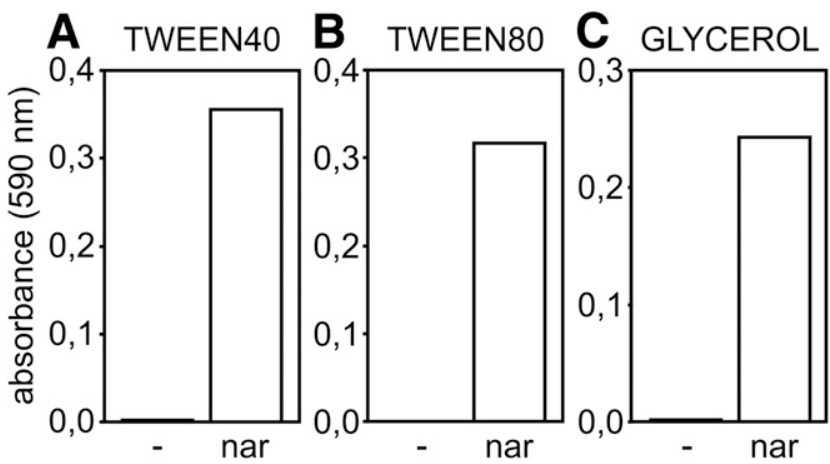

Fig. 6. Cells grown in the presence of naringenin metabolize glycerol and the detergents TWEEN 40 and TWEEN 80 (Sigma-Aldrich, St. Louis). Tetrazolium dye reduction by Bradyrhizobium sp. strain ORS285 cells grown in the absence and presence of $5 \mu \mathrm{M}$ naringenin after 3 days of incubation in GN2 MicroPlates (Biolog, Hayward, CA, U.S.A.) at $28^{\circ} \mathrm{C}$ is shown. Bars represent color absorbance units at $590 \mathrm{~nm}$ due to tetrazolium dye reduction in the presence of A, TWEEN 40; B, TWEEN 80; and C, glycerol.
9D). To analyze whether the isolated fraction was able to induce nod gene expression, we grew an ORS285 nodA-lacZ reporter strain in the presence of the isolated HPLC fraction. Whereas both naringenin and the isolated HPLC fraction stimulated the growth of the reporter strain (data not shown), only naringenin resulted in an increase in the $\beta$-galactosidase activity (Fig. 9E). Mass spectrometric analysis (liquid chromatography/mass
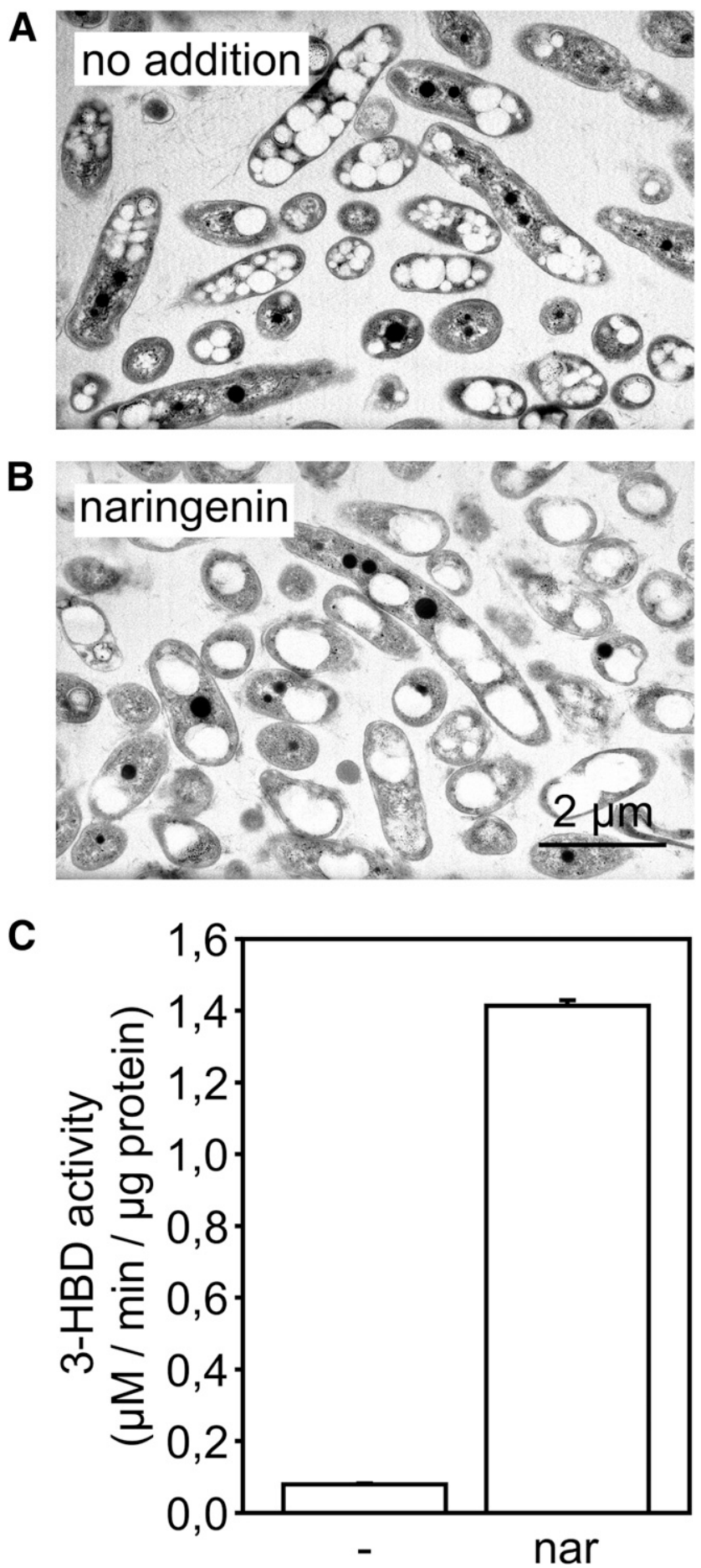

Fig. 7. The effect of $5 \mu \mathrm{M}$ naringenin addition on the cell morphology of Bradyrhizobium sp. strain ORS285. A, Transmission electron microscopy (TEM) image of strain ORS285 cells grown on minimal buffered nodulation medium (BNM). B, TEM image of strain ORS285 cells grown on minimal BNM containing $5 \mu \mathrm{M}$ naringenin. C, 3-Hydroxybutyrate dehydrogenase enzyme activity in cell extracts of strain ORS285 grown for $48 \mathrm{~h}$ in the absence and presence of $5 \mu \mathrm{M}$ naringenin. 
spectrometry [LC/MS]) of this fraction showed that it contained a major product with an $\mathrm{m} / \mathrm{z}$ of 363.0716 (positive ion mode; Supplementary Fig. S5A). Because this molecule is formed after naringenin addition and has an absorbance spectrum with two absorption maxima, which is typical for flavonoids, we hypothesized that the isolated molecule could be a derivative of naringenin. To verify this, we performed LC-MS/MS experiments and compared the obtained masses after fragmentation with the
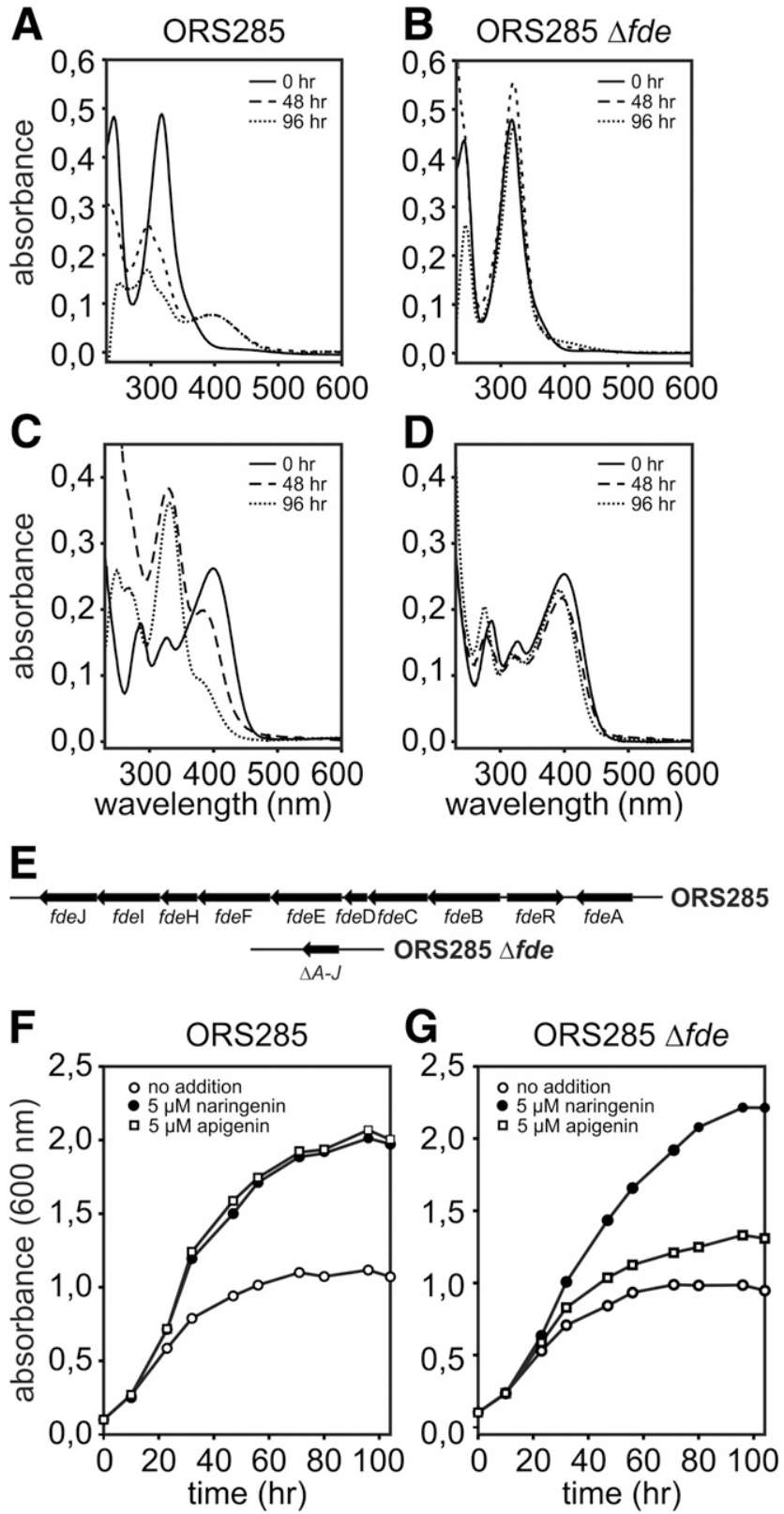

Fig. 8. Bradyrhizobium sp. strain ORS285 degrades the flavonoids naringenin and apigenin via gene products of the fde operon. Absorbance spectra of alkalinized culture supernatant after growth for 0,48 , and $96 \mathrm{~h}$ in the presence of $20 \mu \mathrm{M}$ naringenin for A, wild-type strain ORS285 cells; and B, strain ORS285 $\Delta$ fde cells. Absorbance spectra of alkalinized culture supernatant after growth for 0,48 , and $96 \mathrm{~h}$ in the presence of $20 \mu \mathrm{M}$ apigenin for C, wild-type strain ORS285 cells; and D, strain ORS285 $\Delta f d e$ cells. Absorbance spectra of culture supernatants were corrected using alkalinized buffered nodulation medium as blanc. E, Schematic presentation of the fde operon (open reading frame accession numbers: BRAD285_V2_3671 through 3680) in strains ORS285 and ORS285 $\Delta f d e$ mutant. Representative growth curves in the absence and presence of $5 \mu \mathrm{M}$ naringenin and apigenin, respectively, for F, wild-type strain ORS285 and G, strain ORS285 $\Delta$ fde mutant cells. calculated masses after predicted fragmentation of naringenin derivatives that fit with the previously determined mass (Supplementary Fig. S6). The obtained data are in agreement with our hypothesis and indicate that the isolated molecule is a hydroxylated and O-methylated form of naringenin. Interestingly, an intensely yellow HPLC fraction with exactly the same characteristics was found in the culture supernatant of strain ORS285
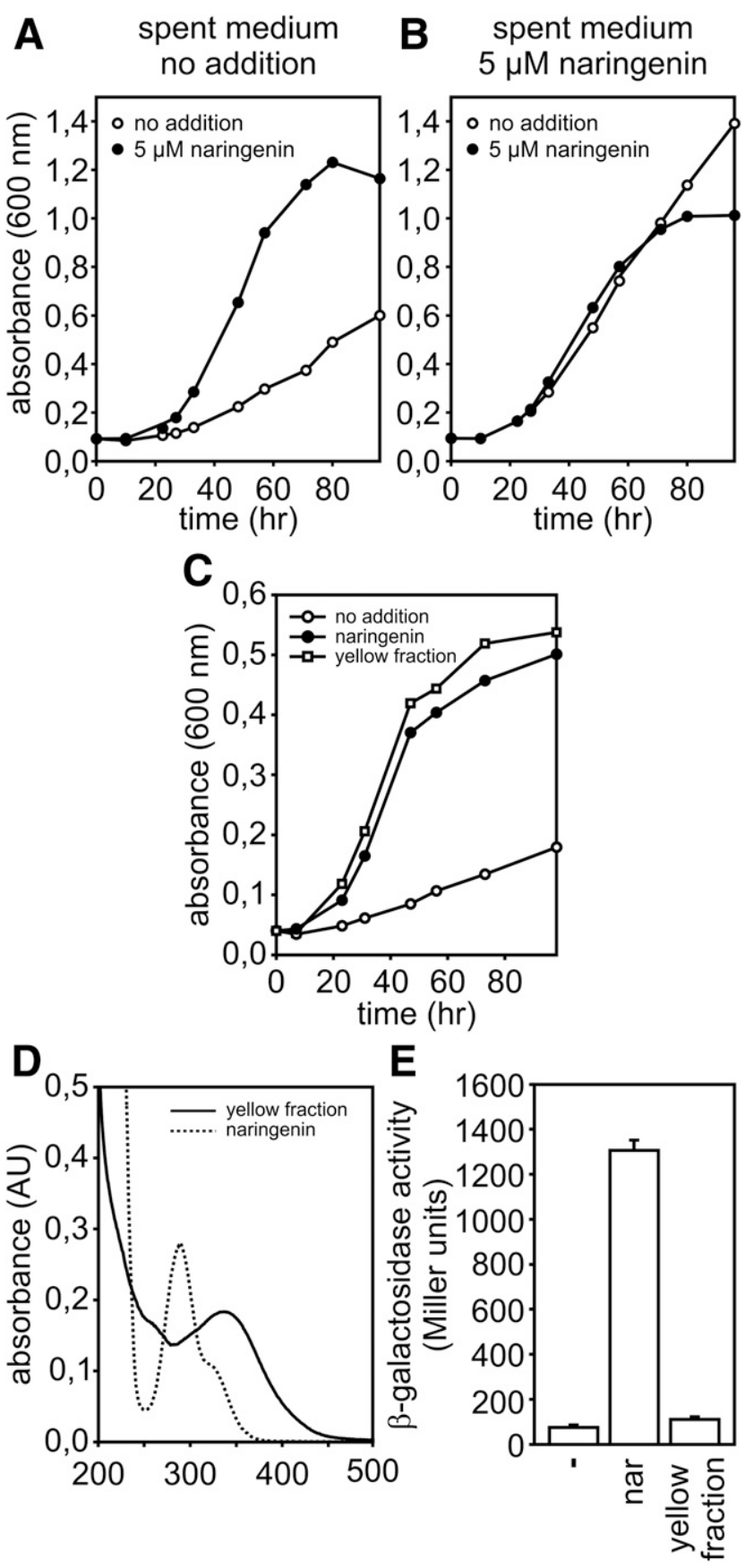

Fig. 9. The culture supernatant of cells grown in the presence of naringenin contains a yellow growth-stimulating molecule. A, Representative growth of Bradyrhizobium sp. strain ORS285 cells in 3x diluted old culture supernatant of strain ORS285 cells. B, Representative growth of strain ORS285 in $3 \times$ diluted old culture supernatant of strain ORS285 cells grown in the presence of $5 \mu \mathrm{M}$ naringenin. $\mathbf{C}$, Representative growth of strain ORS285 in the presence of $5 \mu \mathrm{M}$ naringenin and $15 \mu \mathrm{l}$ intensely yellow high-performance liquid chromatography (HPLC) fraction isolated from the culture supernatant of cells grown in the presence of naringenin. $\mathbf{D}$, Absorbance spectrum of naringenin and yellow HPLC fraction in water. E, $\beta$-Galactosidase activity of the ORS285 nodA-lacZ reporter strain after $24 \mathrm{~h}$ of incubation with $5 \mu \mathrm{M}$ naringenin or $15 \mu \mathrm{l}$ of yellow HPLC fraction. 
$\Delta f d e$ cells grown in the presence of $5 \mu \mathrm{M}$ naringenin. This indicates that the fde operon is not involved in the formation of this yellow growth-stimulating molecule.

\section{DISCUSSION}

Whereas the effect of flavonoids present in root exudates on the expression of nod genes in rhizobia has been well studied, the stimulating effect of certain flavonoids on rhizobial growth is poorly understood. In this report, we studied the growthpromoting effect of the flavonoid naringenin on photosynthetic Bradyrhizobium sp. strain ORS285. Growth stimulation occurred at nanomolar concentrations and was independent of the carbon and nitrogen source in the culture medium (Figs. 2 and 5). Surprisingly, although there is a linear relationship between the initial naringenin concentration and growth-stimulating effect, the final OD and number of CFU with naringenin concentrations $<500 \mathrm{nM}$ are twofold higher than with naringenin concentrations above this concentration (Fig. 2). Concentrationdependent effects of flavonoids on the growth of rhizobia have been observed previously (D'Arcy-Lameta 1986; Hartwig et al. 1991). Many flavonoids have been described as having antimicrobial activity (Cushnie and Lamb 2005). Such activity might explain why higher concentrations of naringenin have a negative impact on the final number of cells in the culture. As shown for other rhizobia (Hartwig et al. 1991), deletion of the nodD1 or nodD2 gene in strain ORS285 had no impact on flavonoiddependent growth stimulation. Thus, whereas both NodD1 and NodD2 are required for naringenin-stimulated expression of the nodA-J operon in strain ORS285 (Nouwen et al. 2016), individually they do not seem to play an important role in the observed growth stimulation.

Phenotypic microarray analysis using GN2 MicroPlates showed that cells grown in the presence of naringenin were able to metabolize glycerol, TWEEN 40, and TWEEN 80 as substrate in contrast to those grown in the absence of naringenin (Fig. 6). TWEEN 40 and TWEEN 80 are nonionic detergents in which an ethoxylated sorbitan is esterified by the fatty acids palmitic acid and oleic acid, respectively. These results suggest that in cells grown in the presence of naringenin glycerol and fatty acid metabolism is activated. Naringenin-dependent stimulated growth also was observed in growth experiments with glycerol as the carbon source (Fig. 5G). To our knowledge, this is the first observation in which the presence of a flavonoid stimulates the growth or drastically reduces the lag phase of a bacterial strain on glycerol. Interestingly, it has been reported that in Rhizobium leguminosarum an operon $(\mathrm{glp})$ required for growth on glycerol was strongly induced by pea seed exudate (Ding et al. 2012). Unfortunately, in this study the authors did not identify the molecule or molecules in the seed exudate responsible for this induction. Naringenin-dependent activation of glycerol and fatty acid metabolism could help strain ORS285 cells to utilize glycerol, fatty acids, and triacylglycerides present at low concentrations in root exudates. Alternatively, fatty acid metabolism might have been activated due to a membranedisturbing effect of naringenin or to energy reserves mobilized to cope with the known antimicrobial effects of flavonoids. Concerning the former, TEM analysis showed a large difference in the number and size of granule-like structures in the cytoplasm between cells grown in the absence and presence of naringenin (Fig. 7A versus B). In the presence of naringenin, only one or two large granule-like structures were found in the cytoplasm of cells, whereas in its absence the cytoplasm was densely packed with numerous small granules. These granulelike structures resemble PHA granules, which are lipid storage particles, found in rhizobia and many other bacteria. This suggests that in the presence of naringenin, PHA turnover is high, whereas under normal conditions strain ORS285 uses PHA formation to store nutrients. Flavonoid-induced activation of fatty acid metabolism and increased catabolism of stored PHA can have multiple functions. First, via PHA catabolism, energy becomes available that enables rhizobia to rapidly colonize the plant rhizosphere. Second, during PHA catabolism, fatty acid precursors are formed that can be used directly to synthesize the acyl-chain present in NFs, as well as to synthesize phospholipids to cope with the membrane-disturbing effects of flavonoids and other stresses during the infection process. In Ensifer meliloti and R. etli, nanomolar concentrations of biotin, a vitamin present in root exudate of alfalfa plants, have been shown to result in the disappearance of PHA granules in the cytoplasm and to stimulate growth (Encarnación et al. 1995; Hofmann et al. 2000). Biotin induces expression of the BdhA enzyme that plays a central role in the degradation of PHA granules (Hofmann et al. 2000). Likewise, in strain ORS285, the BdhA activity is strongly induced in the presence of $5 \mu \mathrm{M}$ naringenin in the growth medium (Fig. 7C). While activation of PHA catabolism appears to be a widespread strategy in rhizobia to stimulate growth; the inducing signal or signals governing this activation remain to be discovered.

In the literature, flavonoid degradation has been postulated as a strategy used by soil bacteria to obtain carbon sources for growth. Strain ORS285 is able to degrade the nod geneinducing flavonoids naringenin and apigenin (Fig. 8A and C). However, when we deleted the operon implicated in naringenin degradation $(f d e)$, naringenin addition still stimulated growth of the bacterium (Fig. 8G). This indicates that under the experimental conditions used, naringenin degradation is not used as an energy source for growth. Remarkably, in contrast to naringenin, apigenin-dependent growth stimulation is almost absent in strain ORS285 $\Delta$ fde cells (Fig. 7G). Previously, it was shown that naringenin is the first intermediate formed in the degradation of apigenin (Schoefer et al. 2003). Thus, the growth-stimulating effect of apigenin is likely due to naringenin formation during its degradation via the enzymes present in the fde operon.

Additional research showed that despite the degradation of naringenin by strain ORS285, a growth-stimulating effect remained present in the growth medium (Fig. 9B). Therefore, we postulated that naringenin addition results in the formation of a molecule or molecules that stimulate growth. Indeed, we were able to isolate a yellow-colored molecule from the growth medium that stimulated the growth of strain ORS285 when added to fresh medium (Fig. 8C). This molecule has an $\mathrm{m} / \mathrm{z}$ of 363.0716 (positive ion mode) that corresponds to a molecule with the formula $\mathrm{C}_{17} \mathrm{H}_{15} \mathrm{O}_{9}$. Based on the formation when naringenin is added, the absorbance spectrum with two absorption maxima that is typical for flavonoids, and the fragmentation pattern obtained in LC-MS/MS experiments, the most likely structure of the isolated molecule is a hydroxylated and O-methylated form of naringenin. However, on the basis of the mass spectrometric data and predicted fragmentation pattern of flavonoids in LC-MS/MS experiments, it is not possible at this step to indicate on which positions the hydroxyl and O-methyl groups are attached to the naringenin core structure.

Interestingly, hydroxylated and methylated forms of naringenin also were found in the culture supernatant of $H$. seropedicae SmR1cells grown in the presence of naringenin (Marin et al. 2016). As with strain ORS285, the formation of these naringenin derivatives was independent of the presence of the fde operon (Marin et al. 2016), and the molecules remained present in the culture medium. One could raise the question of why bacteria modify flavonoids when they are able to degrade them. In general, hydroxylation and methylation of a flavonoid results in a stable and more soluble molecule (Chu et al. 2016). 
For this reason, these modifications have been suggested to be part of a detoxification mechanism to diminish the membranedisturbing effects of flavonoids. At the same time, the increased solubility and stability facilitates the diffusion of the flavonoid into the environment and increases the life span for recognition by bacteria. For this reason, one can also envisage that the hydroxylation and methylation of flavonoids is part of an intercellular communication mechanism that helps in attracting bacteria that are localized further away from the root, to synchronize their metabolism, and to increase the growth rate with the goal of facilitating colonization and infection of host plants. Interestingly, whereas the isolated naringenin derivative was able to stimulate the growth of Bradyrhizobium sp. strain ORS278, it was unable to do so with $B$. diazoefficiens USDA110. Thus, as for the induction of nod gene expression, the growth stimulation seems to be flavonoid and strain specific.

Taken together, this study demonstrates that the roles of flavonoids in bacteria-plant interactions are complex. In rhizobia-legume interactions, it is clear that specific flavonoids induce nod genes via the symbiotic regulator NodD proteins. However, at the same time, nod gene-inducing (and noninducing) flavonoids are degraded and modified into other molecules. These processes, even in standardized model systems, make added flavonoids obtain novel functions that complicate our understanding of effects observed in the rhizobia. Further studies, using strains unable to degrade and modify specific flavonoids might help in understanding the mechanism by which flavonoids have an impact on metabolism and gene expression in rhizobia.

\section{MATERIALS AND METHODS}

\section{Bacterial strains and growth conditions.}

Bradyrhizobium sp. strain ORS285 (Molouba et al. 1999) and derivatives were grown in modified yeast extract mannitol (YM) medium (Giraud et al. 2000) or a minimal buffered nodulation medium (BNM) (Podlešáková et al. 2013) at $28^{\circ} \mathrm{C}$ and $130 \mathrm{rpm}$ in a rotary shaker. For experiments with flavonoids, naringenin and apigenin were added to the culture medium $(20 \mathrm{ml}$ in $50-\mathrm{ml}$ Falcon tubes [Corning Life Sciences, Tewksbury, MA, U.S.A.]) from a $20 \mathrm{mM}$ stock solution in dimethyl sulfoxide. All growth experiments were repeated at least twice. To determine the number of cells in liquid cultures, $10^{1}$ to $10^{8}$ dilutions were made in YM medium, and $50 \mu \mathrm{l}$ of these dilutions was plated on YM plates. After incubation for 5 days at $34^{\circ} \mathrm{C}$, the number of colonies on the plates was counted and used to calculate the number of CFU per milliliter of culture medium. Escherichia coli strains were grown in Luria-Bertani medium at $37^{\circ} \mathrm{C}$. When required, the media were supplemented with kanamycin (50 to $100 \mu \mathrm{g} / \mathrm{ml})$ or cefotaxime $(20 \mu \mathrm{g} / \mathrm{ml})$. All chemicals were purchased from Sigma-Aldrich.

\section{Construction of a strain ORS285 mutant unable to degrade naringenin.}

To construct a deletion of the fde operon in strain ORS285 by crossover PCR, the following sets of primers were used: fdeA_for: 5' CTGCTGGCATGGATCCTAGATGGTCGTGTA GTTGGTGCCGACGTTC 3'; fdeA-rev: 5' CTGAAGCAGTC CGCTCTGGCGACATTG 3'; fdeJ_for 5' CGCCACCGATCC ATCATGGTCGAAG 3'; fdeJ_rev: 5' CTACACGACCATCTA GGATCCATGCCAGCAGCATCGCCGTGTATG 3'. The resulting DNA fragment was cloned into pGEM-T Easy (Promega, Madison, WI, U.S.A.) and transformed into thermocompetent E. coli XL2-Blue cells (Agilent Technologies, Santa Clara, CA, U.S.A.). Correct clones were verified by sequence analysis (Genoscreen, Lille, France). For fdeA-J deletion, the $\Delta f d e A-J$ fragment in pGEM-T Easy was excised with
ApaI-SpeI and ligated into the suicide vector pNPTS139 (Fischer et al. 2002) digested with ApaI-SpeI. The ligation mixture was transformed into $E$. coli XL2-Blue cells, and correct clones were selected via kanamycin resistance (50 $\mu \mathrm{g} / \mathrm{ml}$ ) and subsequent DNA restriction enzyme analysis. Plasmid pNPTS139- $\Delta f d e A-\mathrm{J}$ was transformed into $\mathrm{CaCl}_{2^{-}}$ competent E. coli S17.1 cells. Conjugation, and selection of clones in which the fdeA-J region was deleted, was performed as described previously (Podlešáková et al. 2013).

\section{Phenotypic microarray analysis of cells grown in the presence of naringenin.}

Strain ORS285 was grown in minimal BNM in the absence or presence of $5 \mu \mathrm{M}$ naringenin at $28^{\circ} \mathrm{C}$. At an $\mathrm{OD}_{600}=0.3$, cells were harvested by centrifugation $(15 \mathrm{~min}$ at $4,000 \mathrm{rpm}$ at room temperature). The cell pellet was taken up in assay medium as supplied with the kit until an $\mathrm{OD}_{600}=0.3$ was achieved and distributed in the wells of a GN2 MicroPlate. To prevent a possible induction of metabolic pathways by the tested substrate, chloramphenicol (final concentration of $25 \mu \mathrm{g} / \mathrm{ml}$ ) was added to the assay medium to block further protein synthesis. The plate was incubated at $28^{\circ} \mathrm{C}$, and every $24 \mathrm{~h}$, color development in the wells due to dye reduction (absorbance at $590 \mathrm{~nm}$ ) was measured in a TECAN infinite M200 microplate reader (Tecan, Männedorf, Switzerland).

\section{Bacterial degradation and modification of flavonoids.}

To follow bacterial degradation and modification of flavonoids, strains ORS285 and ORS285 $\Delta f d e$ were grown at $28^{\circ} \mathrm{C}$ in $20-\mathrm{ml}$ cultures of minimal BNM in the absence and presence of $20 \mu \mathrm{M}$ naringenin and $20 \mu \mathrm{M}$ apigenin. At the indicated times, 1 -ml samples were removed from the culture, and the culture supernatant was isolated by centrifugation. After alkalization of the culture medium by addition of $5 \mu \mathrm{l}$ of $1 \mathrm{M} \mathrm{NaOH}$, the absorbance spectrum was measured in a Varian Cary 50 photospectrometer (Agilent Technologies).

\section{TEM.}

Strain ORS285 was grown on BNM agar plates in the absence and presence of $5 \mu \mathrm{M}$ naringenin. After 5 days at $28^{\circ} \mathrm{C}$, bacteria were scraped from the plates and fixed in a $4 \%$ glutaraldehyde, $0.1 \mathrm{M}$ cacodylate buffer ( $\mathrm{pH} 7.2$ ); postfixed in $1 \%$ osmium tetroxide; dehydrated using a series of acetone washes; and embedded in TAAB 812 epon resin. Ultrathin sections $(60 \mathrm{~nm})$ were mounted on collodion carbon-coated copper grids, contrasted using uranyl acetate and lead citrate, and examined at $80 \mathrm{kV}$ with a 100CX II transmission electron microscope (Jeol, Tokyo, Japan).

\section{Isolation of a growth-stimulating factor.}

Strain ORS285 was grown in 1-liter cultures of minimal BNM (3-liter Erlenmeyer flask at $130 \mathrm{rpm}$ ) containing $5 \mu \mathrm{M}$ naringenin. After 3 days at $28^{\circ} \mathrm{C}$, the bacterial culture was centrifuged at $8,000 \mathrm{rpm}$ in a GSA rotor at $4^{\circ} \mathrm{C}$. The culture supernatant was collected and concentrated approximately 12 fold using freeze-drying. The concentrated culture supernatant was filtered through a $0.45-\mu \mathrm{m}$ filter (Merck Millipore, Burlington, MA, U.S.A.) and subsequently passed through a SepPak C18 column (Waters, Milford, MA, U.S.A.) to remove eventual traces of nondegraded naringenin. Of this flowthrough fraction, $25 \mathrm{ml}$ was acidified with phosphoric acid and loaded on an equilibrated Strata X-C column $(500 \mathrm{mg} / 12 \mathrm{ml}$; Phenomenex, Le Pecq, France). After a wash with $10 \mathrm{ml}$ of $0.1 \mathrm{~N}$ $\mathrm{HCl}$, the column was eluted with $10 \mathrm{ml}$ of $100 \%$ methanol. The methanol fraction was dried in a rota-evaporator and dissolved in $500 \mu \mathrm{l}$ of sterile water. To isolate the growth-stimulating factor, $100 \mu \mathrm{l}$ of concentrated Strata X-C eluate was loaded on a Symmetry C18 column ( $5 \mu \mathrm{m}, 4.6 \times 250 \mathrm{~mm}$; Waters $)$ and eluted with 
a linear gradient from 0 to $100 \%$ acetonitrile. The HPLC fraction with an intense yellow color was dried, dissolved in $50 \mu \mathrm{l}$ of water, and analyzed at the mass spectrometry facility of the University of Montpellier. MS experiments were performed using an Alliance 2790 high-performance liquid chromatograph with a photodiode array detector 996 (Waters) coupled to a mass spectrometer (Micromass Q-Tof, Manchester, U.K.) equipped with an electrospray ionization (ESI) source. The highperformance liquid chromatograph was equipped with an ACE reversed-phase column $(\mathrm{C} 18,5 \mu \mathrm{m}, 250 \times 4.6 \mathrm{~mm}$; Waters $)$. The mobile phase consisted of A (water/trifluoroacetic acid: 99.9:0.1, $\mathrm{vol} / \mathrm{vol}$ ) and B (acetonitrile/trifluoroacetic acid, 99.9:0.1, vol/ vol). A gradient starting with 5\% acetonitrile for $4 \mathrm{~min}$ and then increasing to $35 \%$ acetonitrile in $40 \mathrm{~min}$ at $0.7 \mathrm{ml} / \mathrm{min}$ was used for elution. Mass spectra were recorded in positive or negative mode between $50 \mathrm{Da}$ and 1,500 Da. For further identification of the yellow-colored molecule, fragmentation was made by ESI (-) MS/MS with a collision energy of $30 \mathrm{eV}$.

\section{BdhA activity.}

Strain ORS285 cells grown in BNM (100 ml in 500-ml Erlenmeyer flask at $130 \mathrm{rpm}$ and $28^{\circ} \mathrm{C}$ ) in the absence and presence of $5 \mu \mathrm{M}$ naringenin were harvested by centrifugation. After washing the cells with $50 \mathrm{ml}$ of $50 \mathrm{mM}$ TRIS/ $\mathrm{HCl}$ at $\mathrm{pH}$ 8.0 , the cell pellet was taken up into $50 \mathrm{mM}$ TRIS/ $\mathrm{HCl}$ at $\mathrm{pH}$ 8.0 until an $\mathrm{OD}_{600}$ of approximately 20 was achieved. To prepare a cell extract, $100 \mu \mathrm{l}$ of the cell suspension was mixed with lysozyme (final concentration of $10 \mathrm{mg} / \mathrm{ml})$ and TX100 $(0.1 \%$ final concentration). After $30 \mathrm{~min}$ at $37^{\circ} \mathrm{C}, 900 \mu \mathrm{l}$ of $50 \mathrm{mM}$ TRIS/HCl at $\mathrm{pH} 8.0$ was added, and the cells were lysed by passing the mixture eight times through a 26-gauge needle. To measure BdhA activity, $100 \mu \mathrm{l}$ of cell extract was added to a reaction mixture containing $50 \mathrm{mM}$ TRIS/ $\mathrm{HCl}$ at $\mathrm{pH} 8.0$, $1.8 \mathrm{mM} \mathrm{NAD}+$, and $22 \mathrm{mM}$ DL-3-hydroxybutyrate (sodium salt). The conversion of NAD+ to NADH was measured by observing the change in absorbance at $340 \mathrm{~nm}$ using a Varian Cary 50 spectrophotometer. Protein concentration of the cell extract was determined using the Bio-Rad protein assay kit (Bio-Rad Laboratories, Hercules, CA, U.S.A.). BdhA activity is expressed as micromoles per minute per microgram of protein.

\section{LITERATURE CITED}

Arrighi, J.-F., Cartieaux, F., Brown, S. C., Rodier-Goud, M., Boursot, M., Fardoux, J., Patrel, D., Gully, D., Fabre, S., Chaintreuil, C., and Giraud, E. 2012. Aeschynomene evenia, a model plant for studying the molecular genetics of the Nod-independent rhizobium-legume symbiosis. Mol. Plant-Microbe Interact. 25:851-861.

Chu, L. L., Pandey, R. P., Jung, N., Jung, H. J., Kim, E.-H., and Sohng, J. K. 2016. Hydroxylation of diverse flavonoids by CYP450 BM3 variants: Biosynthesis of eriodictyol from naringenin in whole cells and its biological activities. Microb. Cell Fact. 15:135.

Cooper, J. E., Rao, J. R., Everaert, E., and De Cooman, L. 1995 Metabolism of flavonoids by rhizobia. Pages 287-292 in: Nitrogen Fixation: Fundamentals and Applications. I. A. Tikhonovich, N. A. Provorov, V. I. Romanov, and W. E. Newton, eds. Springer Netherlands, Dordrecht, Netherlands.

Cushnie, T. P. T., and Lamb, A. J. 2005. Antimicrobial activity of flavonoids. Int. J. Antimicrob. Agents 26:343-356.

D'Arcy-Lameta, A. 1986. Study of soybean and lentil root exudates. II. Identification of some polyphenolic compounds, relation with plantlet physiology. Plant Soil 92:113-123.

Ding, H., Yip, C. B., Geddes, B. A., Oresnik, I. J., and Hynes, M. F. 2012. Glycerol utilization by Rhizobium leguminosarum requires an $\mathrm{ABC}$ transporter and affects competition for nodulation. Microbiology 158: 1369-1378.

Encarnación, S., Dunn, M., Willms, K., and Mora, J. 1995. Fermentative and aerobic metabolism in Rhizobium etli. J. Bacteriol. 177:3058-3066.

Fischer, B., Rummel, G., Aldridge, P., and Jenal, U. 2002. The FtsH protease is involved in development, stress response and heat shock control in Caulobacter crescentus. Mol. Microbiol. 44:461-478.
Gajendiran, N., and Mahadevan, A. 1988. Utilization of catechin by Rhizobium sp. Plant Soil 108:263-266.

Giraud, E., Hannibal, L., Fardoux, J., Verméglio, A., and Dreyfus, B. 2000. Effect of Bradyrhizobium photosynthesis on stem nodulation of Aeschynomene sensitiva. Proc. Natl. Acad. Sci. U.S.A. 97:14795-14800.

Giraud, E., Moulin, L., Vallenet, D., Barbe, V., Cytryn, E., Avarre, J.-C., Jaubert, M., Simon, D., Cartieaux, F., Prin, Y., Bena, G., Hannibal, L., Fardoux, J., Kojadinovic, M., Vuillet, L., Lajus, A., Cruveiller, S., Rouy, Z., Mangenot, S., Segurens, B., Dossat, C., Franck, W. L., Chang, W.-S., Saunders, E., Bruce, D., Richardson, P., Normand, P., Dreyfus, B., Pignol, D., Stacey, G., Emerich, D., Verméglio, A., Médigue, C., and Sadowsky, M. 2007. Legumes symbioses: Absence of Nod genes in photosynthetic bradyrhizobia. Science 316:1307-1312.

Gough, C., Galera, C., Vasse, J., Webster, G., Cocking, E. C., and Dénarié, J. 1997. Specific flavonoids promote intercellular root colonization of Arabidopsis thaliana by Azorhizobium caulinodans ORS571. Mol. Plant-Microbe Interact. 10:560-570.

Hartwig, U. A., Joseph, C. M., and Phillips, D. A. 1991. Flavonoids released naturally from alfalfa seeds enhance growth rate of Rhizobium meliloti Plant Physiol. 95:797-803.

Hassan, S., and Mathesius, U. 2012. The role of flavonoids in rootrhizosphere signalling: Opportunities and challenges for improving plant-microbe interactions. J. Exp. Bot. 63:3429-3444.

Hofmann, K., Heinz, E. B., Charles, T. C., Hoppert, M., Liebl, W., and Streit, W. R. 2000. Sinorhizobium meliloti strain 1021 bioS and bdhA gene transcriptions are both affected by biotin available in defined medium. FEMS Microbiol. Lett. 182:41-44.

Jain, V., and Gupta, K. 2003. The flavonoid naringenin enhances intercellular colonization of rice roots by Azorhizobium caulinodans. Biol. Fertil. Soils 38:119-123.

Li, F., Hou, B., Chen, L., Yao, Z., and Hong, G. 2008. In vitro observation of the molecular interaction between NodD and its inducer naringenin as monitored by fluorescence resonance energy transfer. Acta Biochim. Biophys. Sin. 40:783-789.

Marin, A. M., de la Torre, J., Marques Oliveira, A. R., Barison, A., Chubatsu, L. S., Monteiro, R. A., de Oliveira Pedrosa, F., Maltempi de Souza, E., Wassem, R., Duque, E., and Ramos, J. L. 2016. Genetic and functional characterization of a novel meta-pathway for degradation of naringenin in Herbaspirillum seropedicae SmR1. Environ. Microbiol. 18:4653-4661.

Marin, A. M., Souza, E. M., Pedrosa, F. O., Souza, L. M., Sassaki, G. L., Baura, V. A., Yates, M. G., Wassem, R., and Monteiro, R. A. 2013. Naringenin degradation by the endophytic diazotroph Herbaspirillum seropedicae SmR1. Microbiology 159:167-175.

Molouba, F., Lorquin, J., Willems, A., Hoste, B., Giraud, E., Dreyfus, B., Gillis, M., de Lajudie, P., and Masson-Boivin, C. 1999. Photosynthetic bradyrhizobia from Aeschynomene spp. are specific to stem-nodulated species and form a separate $16 \mathrm{~S}$ ribosomal DNA restriction fragment length polymorphism group. Appl. Environ. Microbiol. 65:3084-3094.

Nouwen, N., Fardoux, J., and Giraud, E. 2016. NodD1 and NodD2 are not required for the symbiotic interaction of Bradyrhizobium ORS285 with Nod-factor-independent Aeschynomene legumes. PLoS One 11: e0157888.

Panche, A. N., Diwan, A. D., and Chandra, S. R. 2016. Flavonoids: An overview. J. Nutr. Sci. 5:e47.

Podlešáková, K., Fardoux, J., Patrel, D., Bonaldi, K., Novák, O., Strnad, M. Giraud, E., Spíchal, L., and Nouwen, N. 2013. Rhizobial synthesized cytokinins contribute to but are not essential for the symbiotic interaction between photosynthetic bradyrhizobia and Aeschynomene legumes. Mol. Plant-Microbe Interact. 26:1232-1238.

Rao, J. R., and Cooper, J. E. 1994. Rhizobia catabolize nod gene-inducing flavonoids via C-ring fission mechanisms. J. Bacteriol. 176:5409-5413.

Renier, A., Maillet, F., Fardoux, J., Poinsot, V., Giraud, E., and Nouwen, N 2011. Photosynthetic Bradyrhizobium sp. strain ORS285 synthesizes 2O-methylfucosylated lipochitooligosaccharides for nod gene-dependent interaction with Aeschynomene plants. Mol. Plant-Microbe Interact. 24: 1440-1447

Schoefer, L., Mohan, R., Schwiertz, A., Braune, A., and Blaut, M. 2003. Anaerobic degradation of flavonoids by Clostridium orbiscindens. Appl. Environ. Microbiol. 69:5849-5854

Trainer, M. A., and Charles, T. C. 2006. The role of PHB metabolism in the symbiosis of rhizobia with legumes. Appl. Microbiol. Biotechnol. 71: 377-386.

Webster, G., Gough, C., Vasse, J., Batchelor, C. A., O’Callaghan, K. J., Kothari, S. L., Davey, M. R., Dénarié, J., and Cocking, E. C. 1997. Interactions of rhizobia with rice and wheat. Plant Soil 194:115-122.

Yeh, K. C., Peck, M. C., and Long, S. R. 2002. Luteolin and GroESL modulate in vitro activity of NodD. J. Bacteriol. 184:525-530. 